\title{
Diagnostic accuracy of in-house real-time PCR assay for Mycobacterium tuberculosis: a systematic review and meta-analysis
}

\author{
Zhenhong $\mathrm{Wei}^{1 \dagger}$, Xiaoping Zhang ${ }^{1 \dagger}$, Chaojun $\mathrm{Wei}^{2 \dagger}$, Liang $\mathrm{Yao}^{3,4}$, Yonghong $\mathrm{Li}^{2}$, Xiaojing Zhang ${ }^{1}$, Hui Xư ${ }^{2}$,

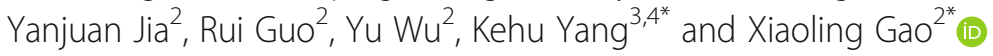

\begin{abstract}
Background: In recent years, studies on the diagnostic accuracy of in-house real-time PCR (hRT-PCR) assay for the detection of Mycobacterium tuberculosis (Mtb) have been reported with unignorable discrepancies. To assess the overall accuracy of the hRT-PCR assay for Mtb diagnosis in different samples for individuals with active pulmonary and extra-pulmonary Mtb infection, a systematic review and meta-analysis were performed.

Methods: The PUBMED, EMBASE, Web of Science, and Cochrane databases were searched up to June 2017 for eligible studies that estimated diagnostic sensitivity and specificity with the hRT-PCR assay in respiratory and nonrespiratory samples in pulmonary and extra-pulmonary Mtb infection patients, with Mtb culture as the reference standard. Bivariate random effect models were used to provide pooled estimation of diagnostic accuracy. Further, subgroup and meta-regression analyses were performed to explore sources of heterogeneity. The risk of bias was assessed by the QUADAS-2 tool.

Results: Of the 3589 candidate studies, 18 eligible studies met our inclusion criteria. Compared to Mtb culture data, the pooled sensitivity and specificity were 0.96 and 0.92, respectively. The diagnostic odds ratio (DOR) was 192.96 (95\% Cl 68.46, 543.90), and the area under the summary ROC curve (AUC) was 0.9791. There was significant heterogeneity in sensitivity and specificity among the enrolled studies $(p<0.001)$. The studies with high-quality assessment and application of respiratory specimen were associated with better accuracy.
\end{abstract}

Conclusions: In low-income/high-burden settings, our results suggested that the hRT-PCR assay could be a useful test for the diagnosis of TB with high sensitivity and specificity.

Keywords: Tuberculosis, Laboratory diagnosis, In-house real-time PCR, Meta-analysis, Systematic review

\section{Background}

Tuberculosis (TB) remains a major public health problem worldwide. In 2015, 10.4 million new cases of TB were reported. Approximately 1.4 million people died of the disease, and there were an additional 0.4 million deaths from co-infected with HIV [1]. Rapid diagnosis

\footnotetext{
* Correspondence: kehuyangebm2006@126.com; gaoxl008@hotmail.com 'Zhenhong Wei, Xiaoping Zhang and Chaojun Wei contributed equally to this work.

${ }^{3}$ The Institute of Clinical Study and Evidence-Based Medicine, Gansu Provincial Hospital, Lanzhou 730000, Gansu, China

${ }^{2}$ The Institute of Clinical Research and Translational Medicine, Gansu Provincial Hospital, No. 204, Donggang street, Chengguan district, Lanzhou 730000, Gansu, China

Full list of author information is available at the end of the article
}

and treatment are pivotal for the effective control of TB in clinical practice [2]. Acid-fast staining and $M t b$ culture are classical $M t b$ diagnosis techniques. The acid-fast stain lacks sensitivity, and the culture requires several weeks for incubation [3, 4]. The inherent limitations make it difficult for them to meet the requirement for early diagnostics [5].

Nucleic acid amplification tests (NAATs), such as polymerase chain reaction (PCR), which was developed in 1983, are now a common tool for the rapid diagnosis of many infectious diseases, including TB [6]. To date, some commercial tests, including COBAS TaqMan, Xpert MTB/RIF and the Abbott Real-Time MTB assay, have been used for TB diagnosis [7-9]. However, due to

(c) The Author(s). 2019 Open Access This article is distributed under the terms of the Creative Commons Attribution 4.0 International License (http://creativecommons.org/licenses/by/4.0/), which permits unrestricted use, distribution, and reproduction in any medium, provided you give appropriate credit to the original author(s) and the source, provide a link to the Creative Commons license, and indicate if changes were made. The Creative Commons Public Domain Dedication waiver (http://creativecommons.org/publicdomain/zero/1.0/) applies to the data made available in this article, unless otherwise stated. 
the limited infrastructure and medical resources, many clinical laboratories in suburban areas with high TB burden cannot afford these assays [1]. In-house polymerase chain reaction (hPCR) that uses equipment and reagents available from diverse suppliers in competitive markets may be more affordable, feasible, and sustainable than Xpert MTB/RIF [10]. hPCR is thus becoming popular in these areas. Several regions of the mycobacterial genome, such as IS6110 and 16S rDNA, have been used as targets for assays [11-13]. Meta-analysis of previous studies demonstrated that the diagnostic accuracy of hPCR assays was variable and inconsistent compared with commercial tests $[14,15]$. For example, the sensitivity of hPCR for tuberculosis meningitis varied between 0 and $100 \%$ [16]. In recent years, PCR technologies have improved markedly with the development of RT-PCR for the detection of mycobacterial infection [17]. This method has the advantage over conventional PCR in speed, automation, high sensitivity and specificity, and a low risk of cross-contamination [18, 19]. In contrast to the commercial kits, an inexpensive RT-PCR would be particularly popular in regions that are short of medical equipment, such as Brazil, India, China, the Russian Federation, Southeast Asian, South Africa, and East Africa. Medical resources are limited in the majority of these countries [1]. Although recent studies have revealed that RT-PCR assays have good diagnostic performance for $\mathrm{TB}$, there are unignorable discrepancies between their results [10, 20-32]. Moreover, none of the researchers could demonstrate precise diagnostic accuracy due to their limited statistical power. Therefore, by systematic review and meta-analysis, we explored factors associated with heterogeneity as well as diagnostic accuracy of the hRT-PCR assay for TB using data from previous studies.

\section{Methods}

The current meta-analysis was conducted according to the guidelines of the Preferred Reporting Items for Systematic Reviews and Meta-Analyses (PRISMA) statement [33]. Since the study was a systematic review and meta-analysis of published articles, patient consent or approval from the institutional ethics committee was not necessary.

\section{Search strategy}

We searched the following databases: PUBMED, EMBASE, Web of Science and Cochrane Library. All searches were up to date as of June 2017. The search terms used included "tuberculosis", "Mycobacterium tuberculosis", "nucleic acid amplification techniques", "real time PCR", "quantitative real-time polymerase chain reaction", "PCR, quantitative real-time", "quantitative realtime PCR", "real-time PCR, quantitative", "sensitivity and specificity", or "predictive value". In addition, the references of several previously published reviews on NAATs were searched for possible candidate articles.

\section{Study selection}

We included all available studies that reported the assessment of hRT-PCR assay for direct detection of TB. Reasons for studies exclusion were (i) the reference standard was not culture proven $M t b$; (ii) studies performed with other assays other than hRT-PCR assay (in mixed Methods Research, data were analysed for the eligible cases separately); (iii) application of hRT- PCR assay for determining drug resistance; (iv) incomplete data (lacking any of the availability data including truepositive, true-negative, false-positive and false-negative or these variables could not be calculated from the published data) were not extracted; (v) evaluation of hRTPCR assay on animal specimens; and (vi) conference abstracts, letters, case reports, editorials, and reviews without original data were excluded.

Two investigators (LY and YHL) independently screened candidate literature by looking up the title and abstract. Then, the full texts of the potentially relevant articles were carefully read to determine whether they could be included. Disagreements were resolved by consensus between the two investigators.

\section{Data extraction and quality assessment}

Two investigators (LY and YHL) independently extracted accurate information from the ultimately included articles. Disagreements were resolved by consensus between the two investigators. The quality of the included studies was independently estimated by two investigators (HX and RG) using a Revised Tool for the Quality Assessment of Diagnostic Accuracy Studies (QUADAS-2), which consists of seven domains [34]. A study was treated as a high-quality study when it had no domain with a high risk of bias and no domain with high applicability concerns.

\section{Statistical analysis}

Analysis was performed using Meta-Disc (version 1.4) software [35]. We pooled the data with the DerSimonian-Laird random effects model (REM), with the following pooled estimates: sensitivity, specificity, positive likelihood ratio $(\mathrm{LR}+)$, negative likelihood ratio (LR-), and the diagnostic odds ratio (DOR).

Each study in the meta-analysis contributed a pair of numbers: sensitivity and specificity. A summary receiver operating characteristic (SROC) curve was constructed for the hRT-PCR assay [36]. A shoulder-like curve illustrates that the variability between studies may be due to the threshold effect. A non-shoulder-like curve indicates that sensitivity and specificity are not correlated. The overall diagnostic performance of that hRT-PCR assay 
was assessed as the area under the curve (AUC) (an AUC value of $100 \%$ indicates a perfect test, while an AUC of 50\% signifies poor diagnostic accuracy) [37, 38].

Heterogeneity between included studies refers to a high degree of variability in study results. The heterogeneity could be explained by variability in thresholds or differences in test methods and study characteristics. Chi-square and Fisher's exact tests were used to detect statistically significant heterogeneity. Heterogeneity between included studies was evaluated with subgroup (stratified) analysis and meta-regression analysis [39]. In the subgroup analysis, we computed pooled DOR estimates in various strata. The following factors as potential sources of heterogeneity: study design, target sequence, respiratory specimen versus non-respiratory specimen, the distribution of $\mathrm{TB}$, and components of study quality.

The meta-regression model produces relative diagnostic odds ratios (RDOR) as the output [39]. An RDOR is a ratio of two DORs. An RDOR of 1.0 explains that a particular covariate does not affect the overall DOR. An RDOR $>1.0$ explains that studies with a particular characteristic have a higher DOR than studies without this characteristic. For an RDOR $<1.0$, the converse is true.

Finally, since publication bias is an important focus for meta-analyses of diagnostic studies [40], the potential publication bias of included studies was assessed by Deeks's funnel plot (Stata version 12.0; Stata Corp., College Station, TX).

\section{Results}

\section{Study search}

Of the 3589 unique articles, we finally identified 15 eligible articles representing 18 independent studies (Fig. 1). The performance of the hRT-PCR assay in Mtb detection of clinical specimens was evaluated from all included studies with $M t b$ culture as a reference standard. Summary characteristics of the included studies are shown in Table 1. Eleven studies used respiratory specimens, and five used non-respiratory specimens. Two

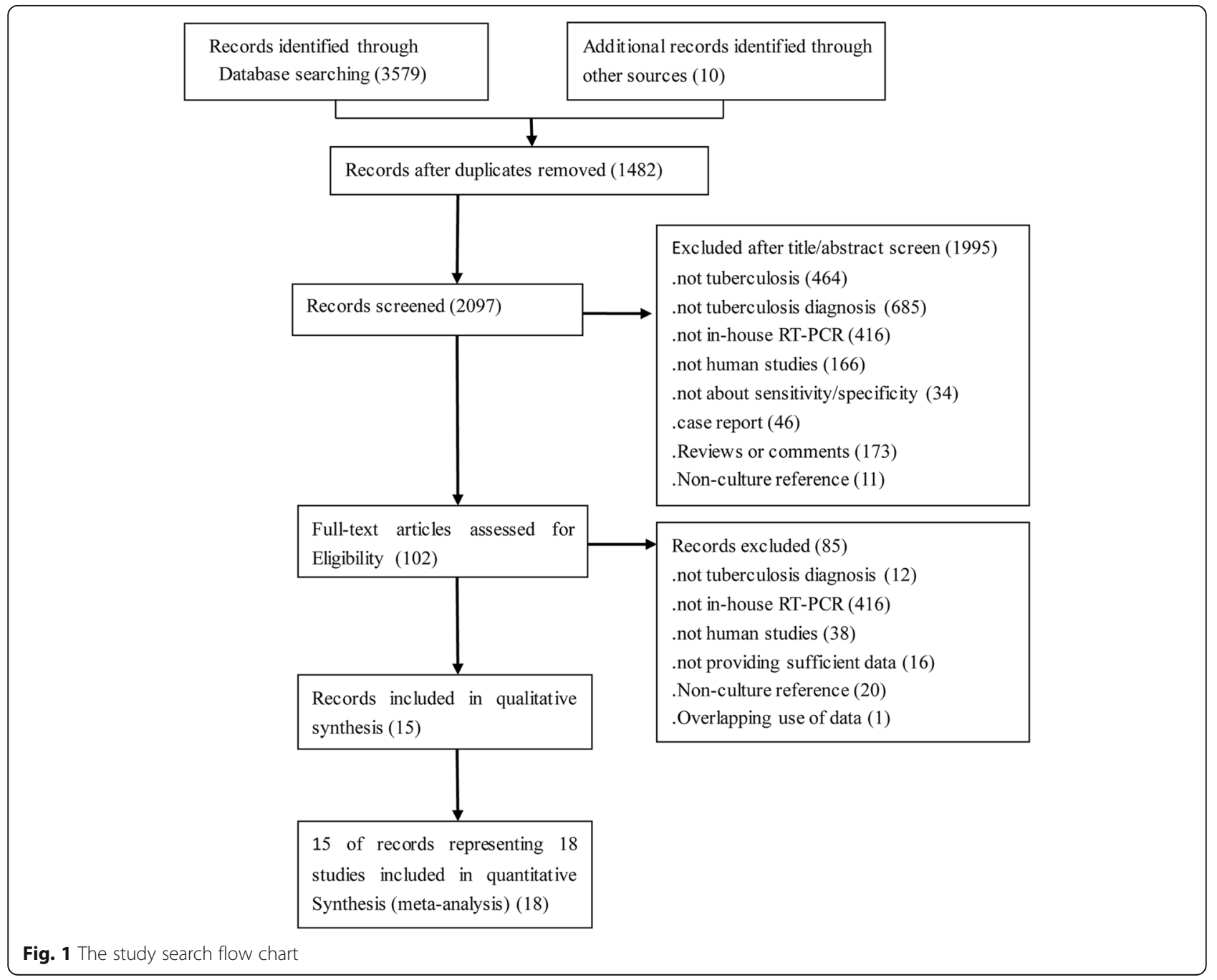




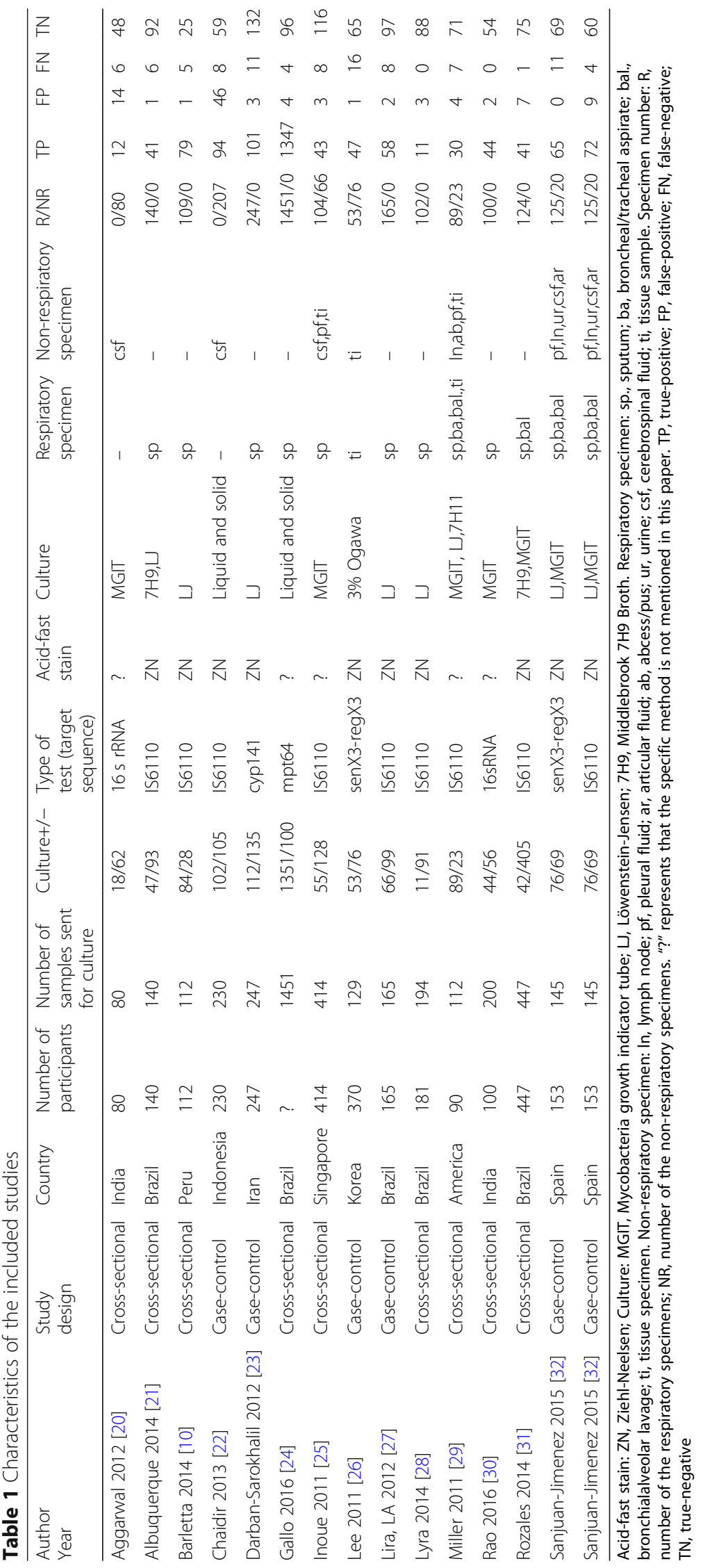


studies focused on patients with HIV-associated TB. Five studies were from Brazil, two were from India, and the remaining studies were from eight different countries. Among them, eight are the high TB burden countries. Eleven studies used IS6110 as an amplification target, and 7 studies used other targets (e.g., mpt64 and senX3regX3). A total of 3281 samples, including 2809 respiratory samples and 472 non-respiratory samples, provided valid results.

\section{Quality evaluation}

We assessed the quality of the studies by QUADAS2. To assess the risk of bias regarding patient selection, three studies were deemed to be of case-control design, which compared diagnosed TB patients to non-TB individuals. Therefore, there was a high risk of bias based on the patient selection method after QUADAS2 assessment. As for "index test" evaluation, four studies failed to illustrate the blind working flow. Given the advanced acknowledgement of the reference test results, the bias could arise from the interpretation of the index test. These studies had an unclear risk of bias on the index test. For the reference standard, two studies did not provide sufficient description concerning the reference test results. Therefore, these studies were considered to have an unclear risk of bias on the reference test. No other domain had a high risk of bias or a high applicability concern (Fig. 2).

\section{Diagnostic accuracy of hRT-PCR assay}

When all 18 studies using the hRT-PCR assay were evaluated together, the overall sensitivity and specificity estimates were 0.96 (95\% CI $0.95,0.96)$ and 0.92 (95\% CI $0.90,0.93)$, respectively. The sensitivity and specificity of all studies are shown in the forest plot (Fig. 3a, b). The overall LR+ was 16.90 (95\% CI 7.22, 39.56), and LR- was 0.11 (95\% CI 0.06, 0.18). The pooled DOR was 192.96 (95\% CI 68.46, 543.90). Heterogeneity was detected by chi-square analysis in the summary results. All measurements showed high heterogeneity $(p<0.001$ for the test of heterogeneity). The accuracy was measured, and their corresponding chi-square test was applied to statistically analyse heterogeneity (Table 2). The overall accuracy of the hRT-PCR assay in a summary receiver operating characteristic (SROC) curve is displayed in Fig. 4, and the curve displayed a trade-off between sensitivity and specificity. The area under the SROC curve (AUC) was 0.9791, indicating a highly diagnostic accuracy. Overall, significant heterogeneity in sensitivity and specificity deserves more attention in the clinical applications of the hRT-PCR assay in TB detection.

\section{Exploration of heterogeneity}

Heterogeneity is an important concern on diagnostic meta-analysis. The threshold effect, method differences

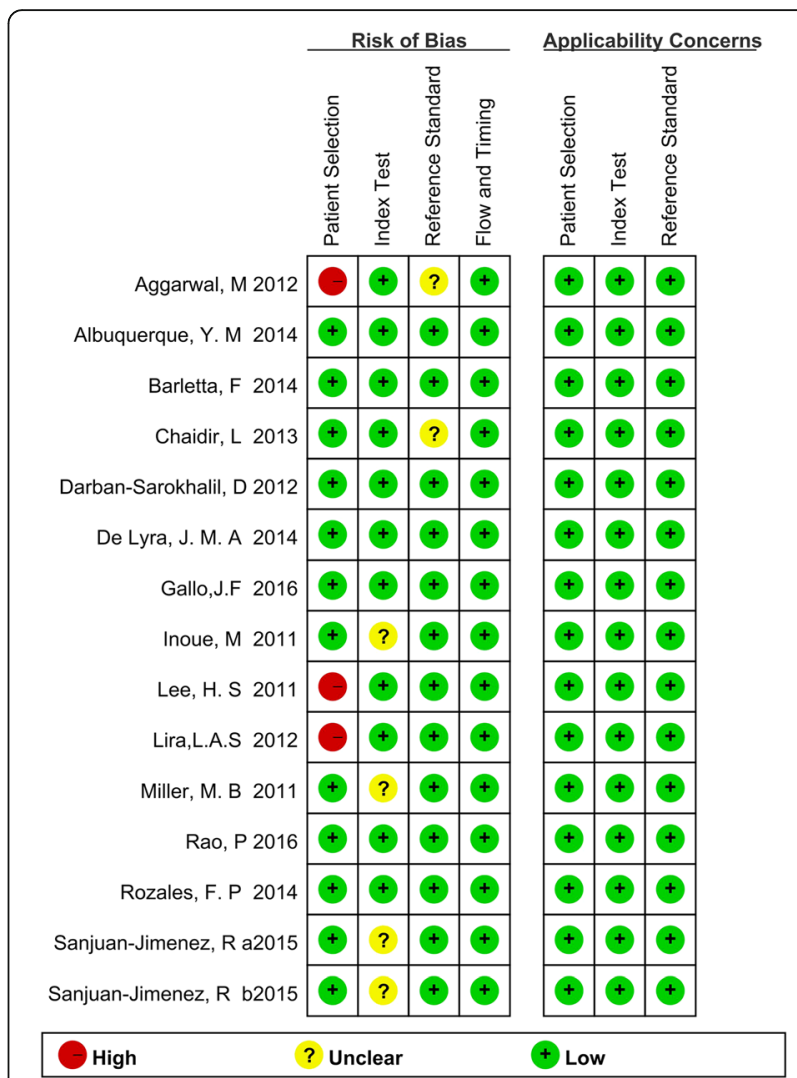

Fig. 2 Summary of methodological quality of studies according to the QUADAS-2 (Quality Assessment of Diagnostic Accuracy Studies2) tool. High-quality study: a study that had no domain with a high risk of bias and no domain with high applicability concerns; medium/moderate-quality study: a study that had domain with an unclear risk of bias or domain with unclear applicability concerns; low-quality study: a study that had a domain with a high risk of bias and domain with high applicability concerns

and study characteristics may lead to the variability. The SROC curve with studies was weighted by their inverse variance, as shown in Fig. 4. The non-shoulder-like curve indicated no threshold effect in the current metaanalysis. Furthermore, the Spearman correlation coefficient was 0.147 , and the $p$ value was 0.562 . It illustrated no threshold effect. Subgroup analysis was also used to explore other factors that were associated with heterogeneity by stratifying data into relatively more homogeneous strata. The DOR estimates of the study characteristics are compared in Table 3. The heterogeneity could be explained in some strata, including specimen type, the distribution of TB, and quality of studies. However, even after stratification, the heterogeneity remained in the evaluation of diagnostic accuracy.

We further performed a meta-regression analysis to explain the variation after subgroup analysis. As shown in Table 4, the RDOR was established from the meta-regression analysis using the restricted maximum 
A

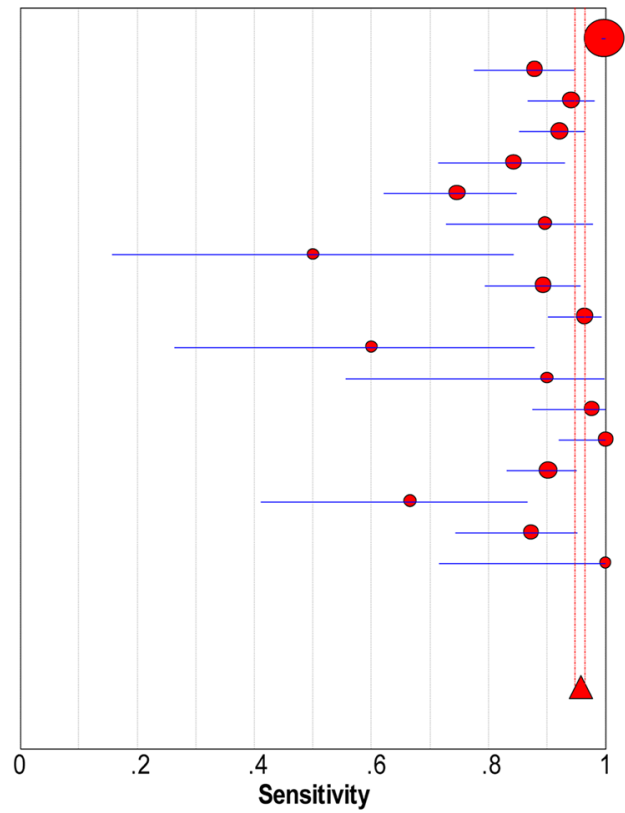

Gallo.J.F

Lira.L.A.S

Barletta. F

Chaidir. L

Inoue. $\mathrm{M}$

Lee. H. S

Miller. M. B

Miller. M. B

Sanjuan-Jimenez. R

Sanjuan-Jimenez. R

Sanjuan-Jimenez. R

Sanjuan-Jimenez. R

Rozales. F. P

Rao. P

Darban-Sarokhalil. D

Aggarwal. M

Albuquerque. Y. M

De Lyra. J. M. A

\section{Sensitivity ( $95 \% \mathrm{Cl}$ )}

$1.00 \quad(0.99-1.00)$

$0.88 \quad(0.78-0.95)$

$0.94 \quad(0.87-0.98)$

$0.92 \quad(0.85-0.97)$

$0.84 \quad(0.71-0.93)$

$0.75 \quad(0.62-0.85)$

$0.90 \quad(0.73-0.98)$

$0.50 \quad(0.16-0.84)$

$0.89 \quad(0.79-0.96)$

$0.97 \quad(0.90-0.99)$

$0.60 \quad(0.26-0.88)$

$0.90 \quad(0.55-1.00)$

$0.98 \quad(0.87-1.00)$

$1.00 \quad(0.92-1.00)$

$0.90 \quad(0.83-0.95)$

$0.67 \quad(0.41-0.87)$

$0.87 \quad(0.74-0.95)$

$1.00(0.72-1.00)$

B

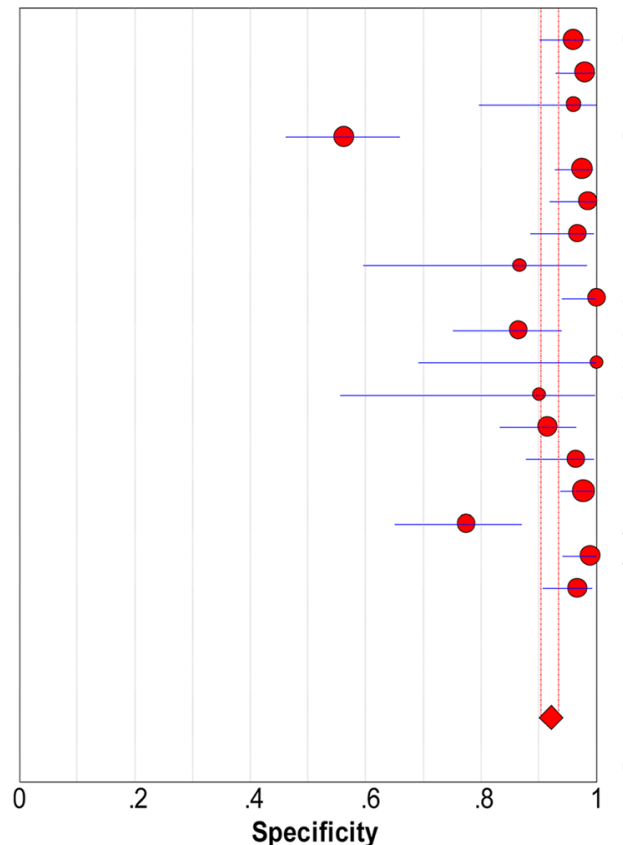

Gallo.J.F

Lira.L.A.S

Barletta. F

Chaidir. L

Inoue. M

Lee. H. S

Miller. M. B

Miller. M. B

Sanjuan-Jimenez. R

Sanjuan-Jimenez. R

Sanjuan-Jimenez. R

Sanjuan-Jimenez. R

Rozales. F. P

Rao. P

Darban-Sarokhalil. D

Aggarwal. M

Albuquerque. Y.M

De Lyra. J. M. A

Specificity $(95 \% \mathrm{Cl})$

$0.96 \quad(0.90-0.99)$

$0.98 \quad(0.93-1.00)$

$0.96 \quad(0.80-1.00)$

$0.56 \quad(0.46-0.66)$

$0.97 \quad(0.93-0.99)$

$0.98 \quad(0.92-1.00)$

$0.97 \quad(0.88-1.00)$

$0.87 \quad(0.60-0.98)$

$1.00(0.94-1.00)$

$0.86(0.75-0.94)$

$1.00 \quad(0.69-1.00)$

$0.90 \quad(0.55-1.00)$

$0.91 \quad(0.83-0.96)$

$0.96 \quad(0.88-1.00)$

$0.98 \quad(0.94-1.00)$

$0.77 \quad(0.65-0.87)$

$0.99 \quad(0.94-1.00)$

$0.97 \quad(0.91-0.99)$

Pooled Specificity $=0.92(0.90$ to 0.93$)$

Chi-square $=172.20 ; \mathrm{df}=17(\mathrm{p}=0.0000)$

Specificity

Fig. 3 Forest plot of specificity and sensitivity estimates. a Forest plot of sensitivity estimates and 95\% confidence intervals (Cl). The point estimates of sensitivity from each study are shown as solid circles. Error bars are $95 \% \mathrm{Cl}$. Circles are proportional to study size. The pooled estimate is denoted by the diamond at the bottom. b Forest plot of specificity estimates and $95 \% \mathrm{Cl}$. The point estimates of specificity from each study are shown as solid circles. Error bars are $95 \% \mathrm{Cl}$. Circles are proportional to study size. The pooled estimate is denoted by the diamond at the bottom

likelihood (REML) method to measure between-study variance. Studies with respiratory specimens produced RDOR values that were significantly higher than those used non-respiratory specimens or both specimens.
Studies with a high-quality level produced RDOR that were significantly higher than those with medium quality levels or low-quality levels. The distribution of TB displayed a slightly higher RDOR but no statistical 
Table 2 Pooled Summary Estimates of All Studies

\begin{tabular}{lll}
\hline Accuracy Measure & $\begin{array}{l}\text { Pooled summary } \\
\text { measure }^{a}(95 \% \mathrm{Cl})\end{array}$ & $\begin{array}{l}\text { P value for } \\
\text { heterogeneity }^{\text {b }}\end{array}$ \\
\hline Sensitivity & $0.96(0.95-0.96)$ & $<0.001$ \\
Specificity & $0.92(0.90-0.93)$ & $<0.001$ \\
Positive Likelihood Ratio (LR+) & $16.90(7.22-39.56)$ & $<0.001$ \\
Negative Likelihood Ratio (LR-) & $0.11(0.06-0.18)$ & $<0.001$ \\
Diagnostic Odds Ratio (DOR) & $192.96(68.46-543.90)$ & $<0.001$ \\
\hline
\end{tabular}

Random effects model

${ }^{\mathrm{b}} \mathrm{Chi}$-square or Fisher's exact test for heterogeneity

significance in the final regression model. Study design and target sequence did not produce a significant RDOR, indicating that the use of any study design and target sequence did not substantially affect diagnostic accuracy. Therefore, specimen types and quality of studies may affect accuracy heterogeneity. Evaluation of the Deeks' $(p=0.11)$ test did not show evidence of publication bias. Furthermore, the funnel plot did not display the presence of asymmetry (Fig. 5).

\section{Discussion}

\section{Principal findings}

We summarized the evidence on the accuracy of the hRT-PCR assay for the diagnosis of TB and performed a meta-regression analysis to explore factors involved in in-house RT-PCR assay performance. This meta-analysis included 18 independent studies with a total of $97 \%$ AUC, indicating that the hRT-PCR assay for TB detection was useful in rapidly identifying $\mathrm{TB}$ cases and that negative data guaranteed the certainty for ruling out active TB. Since there is significant performance heterogeneity in our recruited studies, subgroup and metaregression analysis indicated that the use of respiratory specimens and studies with high quality were associated with better diagnostic accuracy of hRT-PCR.

\section{Clinical implications}

Even though the meta-analysis shows the power in evaluating the overall diagnostic accuracy of hRT-PCR, more caution is necessary to determine clinical accuracy due to significant heterogeneity. Previous meta-analyses $[14,16,41]$ did not fully interpret the cause of heterogeneity found in hPCR results across studies. Our results showed that respiratory specimens and high-quality design were associated with better diagnostic accuracy of the hRT-PCR assay, which was consistent with a recent meta-analysis of the Xpert MTB/RIF PCR assay for the diagnosis of extra-pulmonary TB. There was a performance difference in the specimen site, with low sensitivity in pleural fluid (37\%) and cerebrospinal fluid samples (69\%) [42]. This finding was not surprising given the

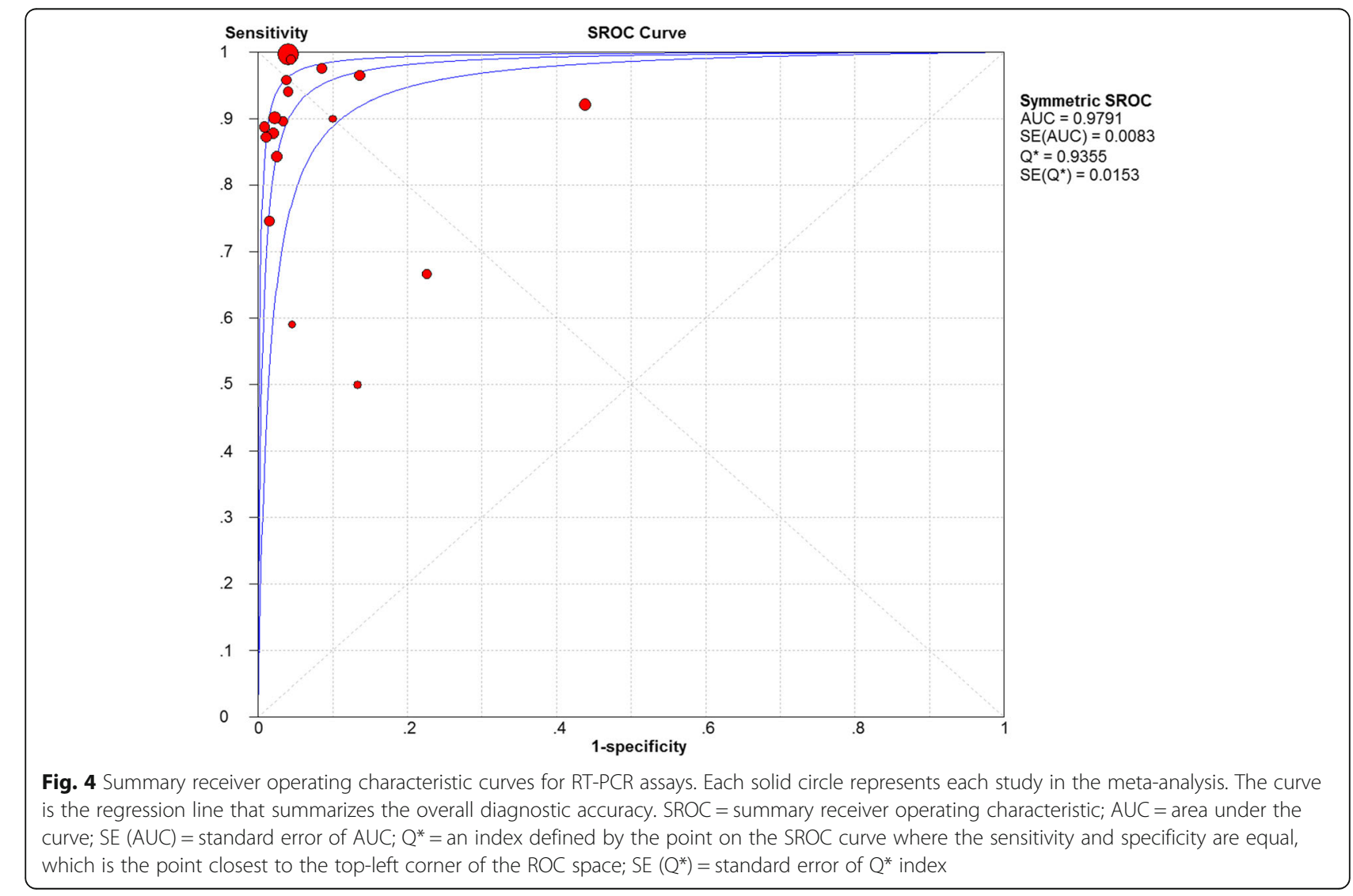


Table 3 Stratified analyses for the evaluation of heterogeneity in studies with real-time PCR assay

\begin{tabular}{|c|c|c|c|}
\hline Subgroup (Number of studies) & Summary diagnostic odds ratio $(95 \% \mathrm{Cl})^{\mathrm{a}}$ & $\mathrm{Chi}^{2}$ square test of heterogeneity & $P$ value for heterogeneity ${ }^{b}$ \\
\hline \multicolumn{4}{|l|}{ Study design } \\
\hline Cross-sectional (10) & $403.18(120.05-1354.05)$ & 36.66 & $<0.001$ \\
\hline Case-control (8) & 73.86 (20.40-267.48) & 34.01 & $<0.001$ \\
\hline \multicolumn{4}{|l|}{ Target sequence } \\
\hline IS6110 (11) & $144.74(51.24-408.86)$ & 39.39 & $<0.001$ \\
\hline Other target (7) & $297.17(30.22-2921.73)$ & 66.27 & $<0.001$ \\
\hline \multicolumn{4}{|l|}{ Specimen type } \\
\hline Respiratory (11) & $598.12(269.12-1329.32)$ & 19.09 & 0.039 \\
\hline Non-respiratory (5) & $12.39(6.67-22.73)$ & 3.57 & 0.468 \\
\hline Both (2) & $202.47(64.68-633.83)$ & 0.00 & 0.944 \\
\hline \multicolumn{4}{|l|}{ Region of study } \\
\hline TB high-burden country (8) & $281.86(37.69-2107.75)$ & 90.46 & $<0.001$ \\
\hline Other country (10) & $160.73(72.80-354.83)$ & 15.17 & 0.086 \\
\hline \multicolumn{4}{|l|}{ Quality of study } \\
\hline High-quality (7) & 926.97 (303.59-2830.38) & 12.83 & 0.046 \\
\hline Medium-quality (8) & 76.77 (22.98-256.50) & 26.65 & $<0.001$ \\
\hline Low-quality (3) & 72.35 (4.47-1170.04) & 19.07 & $<0.001$ \\
\hline
\end{tabular}

${ }^{\mathrm{a}}$ Random effects model

${ }^{b}$ chi-square or Fisher's exact test for heterogeneity; high-quality study: a study that had no domain with a high risk of bias and no domain with high applicability concerns; medium/moderate-quality study: a study that had domain with a unclear risk of bias or domain with unclear applicability concerns; low-quality study: a study that had a domain with a high risk of bias and domain with high applicability concerns

Table 4 Meta-regression analysis to determine sources of heterogeneity

\begin{tabular}{lllll}
\hline Intercept & Coefficient & $P$ value & $\begin{array}{l}\text { Relative } \\
\text { diagnostic } \\
\text { odds ratio } \\
\text { (RDOR) }\end{array}$ & $\begin{array}{l}95 \% \\
\text { confidence } \\
\text { interval }\end{array}$ \\
\hline Intercept & 5.347 & 0.0000 & - & - \\
$\begin{array}{l}\text { Threshold (S) } \\
\text { TB high-burden }\end{array}$ & 0.169 & 0.5382 & - & - \\
$\begin{array}{l}\text { country vs. Other } \\
\text { country }\end{array}$ & 0.756 & 0.3056 & 2.13 & $(0.46 ; 9.96)$ \\
$\begin{array}{l}\text { IS6110 vs. Other } \\
\text { target sequences }\end{array}$ & -0.812 & 0.2266 & 0.44 & $(0.11 ; 1.77)$ \\
$\begin{array}{l}\text { Cross-sectional } \\
\text { design vs. case- } \\
\text { control design }\end{array}$ & -0.759 & 0.5102 & 0.47 & $(0.04 ; 5.45)$ \\
$\begin{array}{l}\text { High-quality level } \\
\text { vs moderate/low- } \\
\text { quality level }\end{array}$ & 1.175 & 0.0272 & 3.24 & $(1.17 ; 9.00)$ \\
$\begin{array}{l}\text { Respiratory specimens } \\
\text { vs non-respiratory } \\
\text { specimens /both }\end{array}$ & 2.262 & 0.0025 & 9.60 & $(2.54 ; 36.25)$ \\
\hline
\end{tabular}

High-quality study: a study that had no domain with a high risk of bias and no domain with high applicability concerns; medium/moderate-quality study: a study that had domain with an unclear risk of bias or domain with unclear applicability concerns; low-quality study: a study that had a domain with a high risk of bias and domain with high applicability concerns paucibacillary nature of these specimens documented in other studies and meta-analysis [14, 16, 42]. The "casecontrol" study design and the IS6110 targeted sequence for hPCR were associated with better accuracy based on previous empirical research and meta-analyses [14, 43$45]$. Some researchers were concerned that the case-control study might overestimate the diagnostic accuracy since it samples patients from the extreme ends of the clinical spectrum (an ideal, "extreme contrast" setting). For example, the sensitivity of a test is evaluated in seriously diseased subjects, and the specificity in healthy individuals [46]. In our meta-analyses, laboratory factors (such as target sequence and amplification technique) weighted more on accuracy than study design features.

The IS6110 gene was widely used for both pulmonary and extra-pulmonary TB diagnosis [13, 47, 48]. Due to its multiple copies in the genome of the $M t b$ complex, PCR might result in better sensitivity [14]. However, our data demonstrated that study design with IS6110 had little impact on diagnostic accuracy. This is possible because RT-PCR used in our enrolled studies carries better advanced technology compared to conventional PCR. RT-PCR uses built-in automated thermocyclers and fluorimeters to monitor PCR reactions in a single tube format in which the reaction processes rapidly and minimizes the risk of contamination from product carryover [49]. Therefore, RT-PCR can provide reliable and repeatable results. 


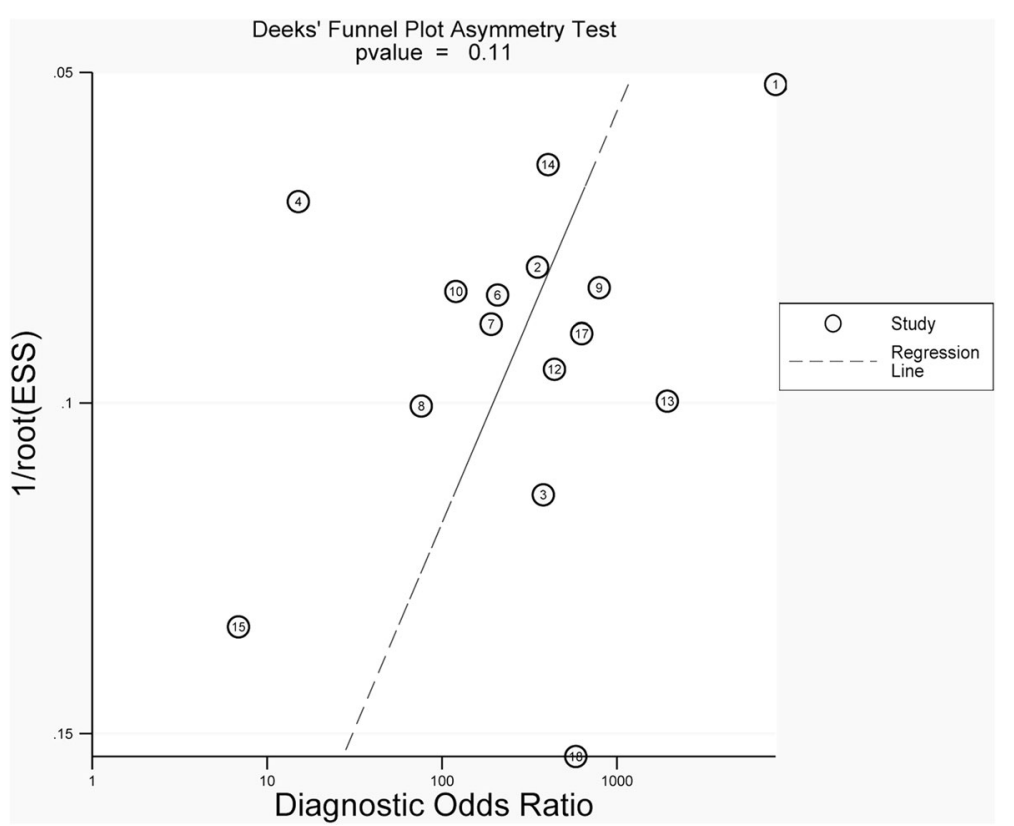

Fig. 5 A Deeks' funnel plot assessment test evaluated the potential publication bias for in-house RT-PCR assays. The plot shows the symmetric distribution of the log of diagnostic odds ratios against the inverse root of effective sample sizes (ESS), indicating the absence of any publication bias

The performance of hRT-PCR was heterogeneous across studies; some patients could have false-positive hRT-PCR results and others false negative. Accuracy is related to the standard/reference assay, TB culture. Reliability is based on clinical diagnosis of TB disease. However, not all recruited studies have evaluated their hRTPCR according to these standards. Caution is highly necessary for the clinical implications and applicability of hRT-PCR. The combination with other clinical information, such as the disease history, family medical records, microscopy screening and histopathology data, is recommended in clinical practice.

\section{Previous meta-analyses of nucleic acid amplification (NAA) test accuracy}

PCR technology is widely used in the diagnosis of infectious diseases. Multiple commercial and in-house NAA techniques have been developed for TB diagnosis [41, 50]. Xpert MTB/RIF, approved by the WHO and the FDA, is a novel, rapid, automated, cartridge-based NAA test that can simultaneously detect $\mathrm{TB}$ and rifampicin resistance directly on untreated sputum [51]. In addition, Xpert MTB/RIF was recommended for the diagnosis of $\mathrm{TB}$ in some special subjects, such as children and patients with certain forms of extra-pulmonary TB. A systematic review showed that Xpert MTB/RIF offered an acceptable sensitivity (62\%) and specificity (98\%) for the diagnosis of pulmonary tuberculosis in children [52]. Compared to hRT-PCR, the main features of Xpert
MTB/RIF are its ability to detect resistance to rifampicin with a simple procedure and high cost. Xpert MTB/RIF may be practical for middle/high income regions. In fact, the majority of low- and middle-income countries, particularly those with limited resources, smear microscopy was still used for TB diagnosis [52]. Therefore, the hRTPCR assay might substitute Xpert MTB/RIF in a low-income setting where multi-drug resistant TB is not prevalent. Another practical assay that might meet the needs of urban areas is loop-mediated isothermal amplification (LAMP) with a commercialized LAMP kit (Loopamp MTBC) [53]. A previous systematic review concerning the LAMP assay included 27 studies [54]; 9 out of the 27 studies evaluated the Loopamp MTBC, and the other 18 evaluated in-house LAMP assays. The summary of sensitivity and specificity for Loopamp MTBC were 80.9 and $96.5 \%$, versus 93.0 and $91.8 \%$ for in-house LAMP assays, respectively. LAMP seems inferior to the RTPCR tests in our analysis. Considering their low cost and simplicity, LAMP assays might be accepted in countries with limited resources.

\section{Limitations of the review}

Our review had some limitations. First, only one study evaluated the diagnostic test accuracy of the hRT-PCR assay for smear status, and only two studies included HIV-positive patients. Therefore, we could not determine the effect of smear and HIV status on the accuracy of the hRT-PCR assay. Second, we only included published 
studies in English, and this could have caused bias in our conclusion. Third, despite the fact that the subgroup analysis and meta-regression analysis could explain part of the observed heterogeneity in accuracy estimates, considerable heterogeneity remained unexplained. Finally, although we searched as many sources as possible, some eligible studies may have been missed.

\section{Conclusion}

In conclusion, based on the meta-analysis using the bivariate model, the diagnostic accuracy of the hRT-PCR assay for TB detection was acceptable. Subgroup and meta-regression analyses were performed, and we found that the diagnostic characteristics were different, depending on the specimen type and quality of the studies. Thus, the hRT-PCR assay, a relatively inexpensive assay compared to other commercial kits, has potential practical value for diagnosing TB, especially in low-income/ high-burden settings, where infrastructures and medical resources are limited.

\section{Additional file}

Additional file 1: Raw data. (XLSX $11 \mathrm{~kb})$

\begin{abstract}
Abbreviations
AUC: Area under the curve; Cl: Confidence interval; DOR: Diagnostic odds ratio; hPCR: In-house polymerase chain reaction; hRT-PCR: In-house real-time polymerase chain reaction; LAMP: Loop-mediated isothermal amplification; Mtb: Mycobacterium tuberculosis; NAATs: Nucleic acid amplification tests; NLR: Negative likelihood ratio; PLR: Positive likelihood; PRISMA: Preferred reporting items for systematic; QUADAS: Quality Assessment of Diagnostic Accuracy Studies; RDOR: Relative diagnostic odds ratios; REM: Random effects model; SROC: Summary receiver operating characteristic; TB: Tuberculosis
\end{abstract}

\section{Acknowledgements}

The authors acknowledge the support of the NSFC Project and the Health Industry Research Project of Gansu.

\section{Authors' contributions \\ $X L G$ and $K H Y$ contributed to the study concept and design, and provided supervision; $L Y$ and $Y H L$ contributed to the literature search, review of the studies, and data extractions; $H X$ and RG contributed to the data analysis and the study quality evaluation; YJJ and XJZ contributed to verifying the statistical analysis and scrutinized data; YW provided expert insight; ZHW, CJW, and XPZ contributed to study design, data analysis, and drafted the manuscript. All authors approved the final version of the manuscript.}

\section{Funding}

The collection and analysis data were funded by the National Natural Science Foundation of China [grant no. 81360264 and 31360508] and the Health Industry Research Project of Gansu Province [grant no. GSWSKY2017-16].

\section{Availability of data and materials}

The dataset supporting the conclusions of this article is included within the article's additional file (Additional file 1).

Ethics approval and consent to participate Not applicable.

\section{Consent for publication}

Not applicable.

\section{Competing interests}

The authors declare that they have no competing interests.

\section{Author details}

'Blood Transfusion Department, Gansu Provincial Hospital, Lanzhou 730000, Gansu, China. ${ }^{2}$ The Institute of Clinical Research and Translational Medicine, Gansu Provincial Hospital, No. 204, Donggang street, Chengguan district, Lanzhou 730000, Gansu, China. ${ }^{3}$ The Institute of Clinical Study and Evidence-Based Medicine, Gansu Provincial Hospital, Lanzhou 730000, Gansu, China. ${ }^{4}$ Evidence-Based Medicine Center and Key Laboratory of Evidence-Based Medicine and Knowledge Translation of Gansu Province, College of Basic Medicine, Lanzhou University, No. 199, Donggang street, Chengguan district, Lanzhou 73000, Gansu, China.

Received: 12 November 2017 Accepted: 10 July 2019

Published online: 08 August 2019

\section{References}

1. Organization WH: Global tuberculosis report 2016.pdf. 2016.

2. Tiwari RP, Hattikudur NS, Bharmal RN, Kartikeyan S, Deshmukh NM, Bisen PS. Modern approaches to a rapid diagnosis of tuberculosis: promises and challenges ahead. Tuberculosis (Edinb). 2007;87(3):193-201.

3. Chagas M, Silva RM, Bazzo ML, Santos JI. The use of polymerase chain reaction for early diagnosis of tuberculosis in mycobacterium tuberculosis culture. Braz J Med Biol Res. 2010;43(6):543-8.

4. Shinu P, Nair A, Singh V, Kumar S, Bareja R. Evaluation of rapid techniques for the detection of mycobacteria in sputum with scanty bacilli or clinically evident, smear negative cases of pulmonary and extra-pulmonary tuberculosis. Mem Inst Oswaldo Cruz. 2011;106(5):620-4.

5. Helb D, Jones M, Story E, Boehme C, Wallace E, Ho K, Kop J, Owens MR, Rodgers R, Banada P, et al. Rapid detection of mycobacterium tuberculosis and rifampin resistance by use of on-demand, near-patient technology. J Clin Microbiol. 2010;48(1):229-37.

6. Diagnostic Standards and Classification of Tuberculosis in Adults and Children. This official statement of the American Thoracic Society and the Centers for Disease Control and Prevention was adopted by the ATS Board of Directors, July 1999. This statement was endorsed by the Council of the Infectious Disease Society of America, September 1999. Am J Respir Crit Care Med. 2000;161(4 Pt 1):1376-95. https://doi.org/10.1164/ ajrccm.161.4.16141.

7. Horita N, Yamamoto M, Sato T, Tsukahara T, Nagakura H, Tashiro K, Shibata Y, Watanabe H, Nagai K, Nakashima K, et al. Sensitivity and specificity of Cobas TaqMan MTB real-time polymerase chain reaction for culture-proven mycobacterium tuberculosis: meta-analysis of 26999 specimens from 17 studies. Sci Rep. 2015;5:18113.

8. Morales-Lopez SE, Yepes JA, Anzola I, Aponte H, Llerena-Polo CR. Paradoxical results of two automated real-time PCR assays in the diagnosis of pleural tuberculosis. Int J Infect Dis. 2017;54:36-8.

9. Park KS, Kim JY, Lee JW, Hwang YY, Jeon K, Koh WJ, Ki CS, Lee NY. Comparison of the Xpert MTB/RIF and Cobas TagMan MTB assays for detection of mycobacterium tuberculosis in respiratory specimens. J Clin Microbiol. 2013:51(10):3225-7.

10. Barletta F, Vandelannoote K, Collantes J, Evans CA, Arevalo J, Rigouts L. Standardization of a TaqMan-based real-time PCR for the detection of mycobacterium tuberculosis-complex in human sputum. Am J Trop Med Hyg. 2014;91(4):709-14.

11. Kox LF, van Leeuwen J, Knijper S, Jansen HM, Kolk AH. PCR assay based on DNA coding for 165 rRNA for detection and identification of mycobacteria in clinical samples. J Clin Microbiol. 1995;33(12):3225-33.

12. Lee BW, Tan JA, Wong SC, Tan CB, Yap HK, Low PS, Chia JN, Tay JS. DNA amplification by the polymerase chain reaction for the rapid diagnosis of tuberculous meningitis. Comparison of protocols involving three mycobacterial DNA sequences, IS6110, 65 kDa antigen, and MPB64. J Neurol Sci. 1994;123(1-2):173-9.

13. Thierry D, Brisson-Noel A, Vincent-Levy-Frebault V, Nguyen S, Guesdon JL, Gicquel B. Characterization of a mycobacterium tuberculosis insertion sequence, IS6110, and its application in diagnosis. J Clin Microbiol. 1990; 28(12):2668-73.

14. Pai M, Flores LL, Hubbard A, Riley LW, Colford JM Jr. Nucleic acid amplification tests in the diagnosis of tuberculous pleuritis: a systematic review and meta-analysis. BMC Infect Dis. 2004;4:6. 
15. Sarmiento OL, Weigle KA, Alexander J, Weber DJ, Miller WC. Assessment by meta-analysis of PCR for diagnosis of smear-negative pulmonary tuberculosis. J Clin Microbiol. 2003;41(7):3233-40.

16. Pai M, Flores LL, Pai N, Hubbard A, Riley LW, Colford JM Jr. Diagnostic accuracy of nucleic acid amplification tests for tuberculous meningitis: a systematic review and meta-analysis. Lancet Infect Dis. 2003;3(10):633-43.

17. Miller N, Cleary T, Kraus G, Young AK, Spruill G, Hnatyszyn HJ. Rapid and specific detection of mycobacterium tuberculosis from acid-fast bacillus smearpositive respiratory specimens and BacT/ALERT MP culture bottles by using fluorogenic probes and real-time PCR. J Clin Microbiol. 2002:40(11):4143-7.

18. Espy MJ, Uhl JR, Sloan LM, Buckwalter SP, Jones MF, Vetter EA, Yao JD Wengenack NL, Rosenblatt JE, Cockerill FR 3rd, et al. Real-time PCR in clinical microbiology: applications for routine laboratory testing. Clin Microbiol Rev. 2006;19(1):165-256.

19. Palomino JC. Nonconventional and new methods in the diagnosis of tuberculosis: feasibility and applicability in the field. Eur Respir J. 2005;26(2): 339-50.

20. Aggarwal M, Aggarwal KC, Nair D. Have we made any headway in the diagnosis of Neurotuberculosis? Place of real time PCR. J Pediatr Infect Dis. 2012;07(04):139-43.

21. Albuquerque YM, Lima AL, Lins AK, Magalhaes M, Magalhaes V. Quantitative real-time PCR (q-PCR) for sputum smear diagnosis of pulmonary tuberculosis among people with HIV/AIDS. Rev Inst Med Trop Sao Paulo. 2014;56(2):139-42.

22. Chaidir L, Ganiem AR, Vander Zanden A, Muhsinin S, Kusumaningrum T, Kusumadewi I, van der Ven A, Alisjahbana B, Parwati I, van Crevel R. Comparison of real time IS6110-PCR, microscopy, and culture for diagnosis of tuberculous meningitis in a cohort of adult patients in Indonesia. PLoS One. 2012;7(12):e52001

23. Darban-Sarokhalil D, Imani Fooladi AA, Maleknejad P, Bameri Z, Aflaki M, Nomanpour B, Yaslianifard S, Modarresi MH, Feizabadi MM. Comparison of smear microscopy, culture, and real-time PCR for quantitative detection of mycobacterium tuberculosis in clinical respiratory specimens. Scand J Infect Dis. 2013;45(4):250-5.

24. Gallo JF, Pinhata JM, Chimara E, Goncalves MG, Fukasawa LO, Oliveira RS. Performance of an in-house real-time polymerase chain reaction for identification of mycobacterium tuberculosis isolates in laboratory routine diagnosis from a high burden setting. Mem Inst Oswaldo Cruz. 2016;111(9):545-50.

25. Inoue M, Tang WY, Wee SY, Barkham T. Audit and improve! Evaluation of a real-time probe-based $P C R$ assay with internal control for the direct detection of mycobacterium tuberculosis complex. Eur J Clin Microbiol Infect Dis. 2011;30(1):131-5

26. Lee HS, Park KU, Park JO, Chang HE, Song J, Choe G. Rapid, sensitive, and specific detection of mycobacterium tuberculosis complex by real-time PCR on paraffin-embedded human tissues. J Mol Diagn. 2011;13(4):390-4.

27. Lira LA, Santos FC, Carvalho MS, Montenegro RA, Lima JF, Schindler HC, Montenegro LM. Evaluation of a IS6110-Taqman real-time PCR assay to detect mycobacterium tuberculosis in sputum samples of patients with pulmonary TB. J Appl Microbiol. 2013;114(4):1103-8.

28. Lyra JM, Maruza M, Verza M, Carneiro MM, Albuquerque Mde F, Rossetti ML, Ximenes R, Braga MC, Lucena-Silva N. Evaluation of four molecular methods for the diagnosis of tuberculosis in pulmonary and blood samples from immunocompromised patients. Mem Inst Oswaldo Cruz. 2014;109(6):805-13.

29. Miller MB, Popowitch EB, Backlund MG, Ager EP. Performance of Xpert MTB/ RIF RUO assay and IS6110 real-time PCR for mycobacterium tuberculosis detection in clinical samples. J Clin Microbiol. 2011:49(10):3458-62.

30. Rao P, Chawla K, Shenoy VP, Mukhopadhyay C. Role of real-time PCR for detection of tuberculosis and drug resistance directly from clinical samples. Indian J Tuberc. 2016;63(3):149-53.

31. Rozales FP, Machado AB, De Paris F, Zavascki AP, Barth AL. PCR to detect mycobacterium tuberculosis in respiratory tract samples: evaluation of clinical data. Epidemiol Infect. 2014;142(7):1517-23.

32. Sanjuan-Jimenez R, Toro-Peinado I, Bermudez P, Colmenero JD, Morata P. Comparative study of a real-time PCR assay targeting senX3-regX3 versus other molecular strategies commonly used in the diagnosis of tuberculosis. PLoS One. 2015;10(11):e0143025.

33. Moher D, Liberati A, Tetzlaff J, Altman DG. Preferred reporting items for systematic reviews and meta-analyses: the PRISMA statement. Int J Surg. 2010;8(5):336-41.

34. Whiting PF, Rutjes AW, Westwood ME, Mallett S, Deeks JJ, Reitsma JB, Leeflang MM, Sterne JA, Bossuyt PM. QUADAS-2: a revised tool for the quality assessment of diagnostic accuracy studies. Ann Intern Med. 2011; 155(8):529-36.

35. Zamora J, Abraira V, Muriel A, Khan K, Coomarasamy A. Meta-DiSc: a software for meta-analysis of test accuracy data. BMC Med Res Methodol. 2006;6:31

36. Littenberg B, Moses LE. Estimating diagnostic accuracy from multiple conflicting reports: a new meta-analytic method. Med Decis Making. 1993; 13(4):313-21.

37. Deeks JJ. Systematic reviews in health care: systematic reviews of evaluations of diagnostic and screening tests. BMJ. 2001;323(7305):157-62.

38. Irwig L, Macaskill P, Glasziou P, Fahey M. Meta-analytic methods for diagnostic test accuracy. J Clin Epidemiol. 1995;48(1):119-30 discussion 131-112.

39. Lijmer JG, Bossuyt PM, Heisterkamp SH. Exploring sources of heterogeneity in systematic reviews of diagnostic tests. Stat Med. 2002;21(11):1525-37.

40. Song F, Khan KS, Dinnes J, Sutton AJ. Asymmetric funnel plots and publication bias in meta-analyses of diagnostic accuracy. Int J Epidemiol. 2002;31(1):88-95.

41. Flores LL, Pai M, Colford JM Jr, Riley LW. In-house nucleic acid amplification tests for the detection of mycobacterium tuberculosis in sputum specimens: meta-analysis and meta-regression. BMC Microbiol. 2005;5:55.

42. Penz E, Boffa J, Roberts DJ, Fisher D, Cooper R, Ronksley PE, James MT. Diagnostic accuracy of the Xpert(R) MTB/RIF assay for extra-pulmonary tuberculosis: a meta-analysis. Int J Tuberc Lung Dis. 2015;19(3):278-84 i-iii.

43. Kocagoz T, Yilmaz E, Ozkara S, Kocagoz S, Hayran M, Sachedeva M, Chambers HF. Detection of mycobacterium tuberculosis in sputum samples by polymerase chain reaction using a simplified procedure. J Clin Microbiol. 1993;31(6):1435-8.

44. Nolte FS, Metchock B, McGowan JE Jr, Edwards A, Okwumabua O, Thurmond C, Mitchell PS, Plikaytis B, Shinnick T. Direct detection of mycobacterium tuberculosis in sputum by polymerase chain reaction and DNA hybridization. J Clin Microbiol. 1993;31(7):1777-82.

45. Noordhoek GT, Kaan JA, Mulder S, Wilke H, Kolk AH. Routine application of the polymerase chain reaction for detection of mycobacterium tuberculosis in clinical samples. J Clin Pathol. 1995:48(9):810-4.

46. Knottnerus JA, van Weel C, Muris JW. Evaluation of diagnostic procedures. BMJ. 2002:324(7335):477-80.

47. Negi SS, Anand R, Pasha ST, Gupta S, Basir SF, Khare S, Lal S. Diagnostic potential of IS6110, 38kDa, 65kDa and 85B sequence-based polymerase chain reaction in the diagnosis of mycobacterium tuberculosis in clinical samples. Indian J Med Microbiol. 2007;25(1):43.

48. van Embden JD, Cave MD, Crawford JT, Dale JW, Eisenach KD, Gicquel B, Hermans P, Martin C, McAdam R, Shinnick TM, et al. Strain identification of mycobacterium tuberculosis by DNA fingerprinting: recommendations for a standardized methodology. J Clin Microbiol. 1993;31(2):406-9.

49. Cockerill FR 3rd. Application of rapid-cycle real-time polymerase chain reaction for diagnostic testing in the clinical microbiology laboratory. Arch Pathol Lab Med. 2003;127(9):1112-20.

50. Ling DI, Flores LL, Riley LW, Pai M. Commercial nucleic-acid amplification tests for diagnosis of pulmonary tuberculosis in respiratory specimens: meta-analysis and meta-regression. PLoS One. 2008;3(2):e1536.

51. Organization WHO. Tuberculosis diagnostic Xpert MTB/RIF test. Geneva: WHO; 2014.

52. Detjen AK, DiNardo AR, Leyden J, Steingart KR, Menzies D, Schiller I, Dendukuri N, Mandalakas AM. Xpert MTB/RIF assay for the diagnosis of pulmonary tuberculosis in children: a systematic review and meta-analysis. Lancet Respir Med. 2015;3(6):451-61.

53. Yu L, Xu Y, Wang F, Yang C, Liu G, Song X. Functional roles of pattern recognition receptors that recognize virus nucleic acids in human adiposederived mesenchymal stem cells. Biomed Res Int. 2016;2016:9872138.

54. Nagai K, Horita N, Yamamoto M, Tsukahara T, Nagakura H, Tashiro K, Shibata Y, Watanabe H, Nakashima K, Ushio R, et al. Diagnostic test accuracy of loop-mediated isothermal amplification assay for mycobacterium tuberculosis: systematic review and meta-analysis. Sci Rep. 2016;6:39090.

\section{Publisher's Note}

Springer Nature remains neutral with regard to jurisdictional claims in published maps and institutional affiliations. 